\title{
Effects of cerebrolysin on nerve growth factor system in the aging rat brain
}

\author{
Mikhail Stepanichev $^{\mathrm{a}, 1}$, Mikhail Onufriev ${ }^{\mathrm{a}, \mathrm{b}, 1}$, Viktor Aniol ${ }^{\mathrm{a}}$, Sofia Freiman ${ }^{\mathrm{a}, \mathrm{b}}$, \\ Hemma Brandstaetter ${ }^{c}$, Stefan Winter ${ }^{\mathrm{c}}$, Natalia Lazareva ${ }^{\mathrm{a}}$, Alla Guekht ${ }^{\mathrm{b}}$ and Natalia Gulyaeva ${ }^{\mathrm{a}, \mathrm{b}, *}$ \\ a'Institute of Higher Nervous Activity and Neurophysiology, Russian Academy of Sciences, Moscow, Russia \\ bMoscow State Budgetary Health Institution "Moscow Research and Clinical Center for Neuropsychiatry" \\ of the Healthcare Department of Moscow, Russia \\ ${ }^{\mathrm{c}}$ Department of Research and Medical, EVER Neuro Pharma GmbH, Unterach, Austria
}

\begin{abstract}
.
Background: Aging is associated with some cognitive decline and enhanced risk of development of neurodegenerative diseases. It is assumed that altered metabolism and functions of neurotrophin systems may underlie these age-related functional and structural modifications. Cerebrolysin ${ }^{\mathrm{TM}}$ (CBL) is a neuropeptide mixture with neurotrophic effects, which is widely used for the treatment of stroke and traumatic brain injury patients. It is also evident that CBL has an overall beneficial effect and a favorable benefit-risk ratio in patients with dementia. However, the effects of CBL on cognition and brain neurotrophin system in normal aging remain obscure.

Objective: The aim of the present study was to examine the age-related modifications of endogenous neurotrophin systems in the brain of male Wistar rats and the effects of CBL on learning and memory as well as the levels neurotrophins and their receptors.

Methods: Old (23-24 months) and young (2-3 months) male Wistar rats were used for the study. A half of animals were subjected to CBL course ( $2.5 \mathrm{ml} / \mathrm{kg}, 20$ i.p. injections). Behavior of rats was studied using the open field test and simple water maze training. The contents of NGF and BDNF were studied using enzyme-linked immunosorbent assay; the expression of neurotrophin receptors was estimated by Western-blot analysis.

Results: CBL treatment did not affect general status, age-related weight changes, general locomotor activity as well as general brain histology. In a water maze task, a minor effect of CBL was observed in old rats at the start of training and no effect on memory retention was found. Aging induced a decrease in neurotrophin receptors TrkA, TrkB, and p75NTR in the neocortex. CBL counteracted effects of aging on neocortical TrkA and p75NTR receptors and decreased expression of proNGF without influencing overall NGF levels. BDNF system was not significantly affected by CBL.

Conclusion: The pro-neuroplastic "antiaging" effects of CBL in the neocortex of old animals were generally related to the NGF rather than the BDNF system.
\end{abstract}

Keywords: Aging, brain, Cerebrolysin, neurotrophin, NGF, BDNF, TrkA, TrkB, p75NTR, neocortex, hippocampus, memory, rat

\section{Introduction}

Aging is a physiological process associated with some cognitive decline and enhanced risk of

\footnotetext{
${ }^{1}$ M.S and M.O. equally contributed to the study.

*Corresponding author: Professor Natalia Gulyaeva, Institute of Higher Nervous Activity and Neurophysiology, Russian Academy of Sciences, 5a Butlerov str., Moscow 117485, Russia. Tel.: +7 495 9524007; Fax: +7 495 9524007; E-mail: nata_gul@yahoo.com.
}

development of neurodegenerative diseases. It is generally assumed that these age-related functional and structural modifications are associated with altered metabolism and functions of neurotrophins (Budni et al., 2015). Neurotrophins are structurally similar growth factors that control cell survival, regulate a wide variety of neural functions and are critically involved in neuroplasticity, memory, and learning in the adult nervous system (see (Bothwell, 2014), for a review). The effects of neurotrophins depend 
on their availability, their affinity of binding to transmembrane receptors Trk and p75NTR, and the downstream signaling events stimulated by receptor activation. Alterations in neurotrophin levels, particularly nerve growth factor (NGF) and brain-derived neurotrophic factor (BDNF), have been implicated in neurodegenerative disorders, such as Alzheimer's disease and Huntington's disease, as well as psychiatric disorders, including depression and substance abuse (Chao et al., 2006). Specifically, NGF secreted by cells in the cortex and hippocampus and its interaction with high-affinity (TrkA) and low-affinity (p75NTR) receptors, are involved in survival of basal forebrain cholinergic neurons. Dysfunction of NGF and its receptors has been suggested to underlie the selective degeneration of these neurons in Alzheimer's disease (Cuello et al., 2007; Niewiadomska et al., 2011).

Published data on the age-related changes of neurotrophins and their receptors in rat brain reveal a surprising discrepancy between the results of different research groups. Inconsistencies might originate from differences in methods, rat strains, brain regions, gender, or in experimental designs (including small number of animals in a group.). It is likely that a combination of these factors contributes to the variance of study results. In addition, the conditions of animals' handling and stress factors should not be neglected. For instance, in the hippocampus of male SpragueDawley rats, the up/down-regulation of BDNF and TrkB were affected by aging and the stimulus paradigm (acute or chronic stress) (Shi et al., 2010).

It is obviously considered that depletion of neurotrophins (accompanied by an increase in their respective pro-forms) and their active receptors occurs with progressive aging. However, reports remain contradictory. For example, Bimonte-Nelson et al. (2008) demonstrated region- and genderdependent changes of NGF and BDNF in the brain of aged Fischer 344 rats and concluded that profound changes in neurotrophin protein levels can occur within only a few months. This study contradicts to the data of Crutcher and Weingartner (1991): this group was unable to show any decline in NGF levels with age or to find any differences between male and female rats.

Similarly, Lapchak et al. (1993) used Northern blot analysis and confirmed that neither bdnf mRNA nor trkB mRNA levels changed with age in the hippocampus of male Fischer 344 rats.

According to Perovic et al. (2013), proNGF levels increased during aging in the neocortex of Wistar rats; mature NGF gradually decreased in the cortex and, in 24-month-old animals, it was 30\% lower when compared to adult 6-month-old rats. The BDNF expression did not change during aging, while proBDNF accumulated in the hippocampus of aged rats. However, there are some concerns about this study, one of them related to small number of animals in the groups.

It has been supposed that modulation of neurotrophic factors may be part of the therapeutic strategy for neurological disorders, in particular dementia, stroke and traumatic brain injury. The same may be true for aging and age-related disturbances in CNS functions. Ukraintseva et al. (2004) noticed that during the last decades physicians in many developed countries have successfully prescribed several medicines to cure various symptoms of senescence, though the influence of such medicines on healthy elderly humans practically has not been studied. Cerebrolysin ${ }^{\mathrm{TM}}$ (CBL, EVER Neuro Pharma GmbH, Austria) is one of the most common nootropic drugs indicated for clinical use in stroke, traumatic brain injury and dementia. The therapeutic effects of CBL are thought to be associated with its potential neurotrophic activity (Masliah \& Dlez-Tejedor, 2012). Available data from human clinical trials and experimental animal studies indicate that treatments with CBL improve learning, memory, brain metabolism and capacity. This means that modern medicine already has "antiaging" treatments at its disposal (Ukraintseva et al., 2004). However, the influence of such treatments on the aged brain has been poorly studied. Indeed, though effects of CBL on aged brain are of obvious clinical significance, these studies are surprisingly rare.

The aim of the present study was to investigate age-related alterations in the neurotrophin system and the effects of CBL course on the endogenous neurotrophin system in the brain of old rats (23-24 months) representative for age related alterations as compared to young animals (2-3 months).

\section{Methods}

\subsection{Animals}

All animals were supplied by Stolbovaya Animal Farm (Moscow region, Russia). Male Wistar rats came to the institutional animal facility at the age of 2-3 months and were housed 5 per a cage under $12: 12 \mathrm{~h}$ light/dark cycle until the age of 6 months. 
Then, they were reassigned into small groups of 2-3 rats and housed until the start of experimental treatment. Food (extruded combined foraging for rats, Laboratorkorm, Russia) and fresh water were available ad libitum.

At the age of $2-3$ or $23-24$ months, the animals were divided into experimental groups treated with CBL (provided by Ever Neuro Pharma, Austria) at a dose of $2.5 \mathrm{ml} / \mathrm{kg}$ or equal volume of Vehicle (sterile isotonic saline solution, Mosfarm, Russia). All injections were performed daily, twenty injections in total for a period of 28 days with four treatment cycles of five days and a pause of two days in between. Prior to the treatment, the animals were subjected to the open field (OF) test in order to measure the initial level of locomotor and exploratory activity, and these data were used for assigning them to the experimental groups. Data from the test were classified according to animal distance traveled and rearing from higher to lower indices, and then, the animals were assigned to one of two groups stepwise. This allowed to complete two groups (within each age) with similar levels of locomotor and exploratory activity observed in the OF test. All experiments were undertaken in accordance with the ethical principles stated in the European directive (86/609/EC) and were approved by the Ethical Committee of the Institute of Higher Nervous Activity and Neurophysiology of the Russian Academy of Sciences. All behavioral and biochemical testings were conducted blind.

\subsection{Open field test}

Open field (OF) test was performed as described elsewhere (Kelley, 1993) in a quiet room. We used a circular arena $120 \mathrm{~cm}$ in diameter painted in white. The floor of the arena was divided into squares of $20 \times 20 \mathrm{~cm}$. The arena was lighted $(100 \mathrm{~lx})$ with a bulb located over the center. The animal was put in the center of the arena and tested for $5 \mathrm{~min}$. Animal behavior in the OF was recorded and analyzed by a scientist who was blinded to the treatment. The following behavioral indices were counted: latency of the first moving out of the arena center, number of squares crossed, number of rearings, number of entries into the central area, number of defecation boli. OF test was performed 2 days prior to the start of the treatment. The data of preliminary testing were used for randomization of the animals into experimental groups.

\subsection{Place learning in a water-maze}

Long-term memory was examined using the successive training procedure of place learning in a water-maze, between 1st and 5th day after the end of course administration of Vehicle or CBL. The water-maze consisted of a gray metal rectangular pool $(100 \times 60 \times 35 \mathrm{~cm})$, filled with water to a height of $27 \mathrm{~cm}$ (Maurice et al., 1996). Milk powder was used to render the water opaque. The water temperature was maintained at $22 \pm 1^{\circ} \mathrm{C}$ with a bath heater, used before sessions. A transparent plexiglass platform $(15 \times 15 \mathrm{~cm})$ was fixed in the middle of the west side of the pool, $1 \mathrm{~cm}$ below the water surface. On days 1 and 2, animals in groups of 14-15 were assessed for consecutive training trials. Each rat was placed at the northeast or southeast corner of the pool, facing the wall, and the latency to find the platform was recorded up to $60 \mathrm{~s}$. Animals were allowed to remain on the platform for $20 \mathrm{~s}$, and then, removed from the maze to their home cages. If the rat did not find the platform within $60 \mathrm{~s}$, the latency was assigned as $65 \mathrm{~s}$, and the rat was manually placed on the platform, left for $20 \mathrm{~s}$, and returned to its original cage. The escape latency (EL), i.e., the time spent to reach the platform during the 60-s session, was recorded. Each group of animals was run in this manner for a total of 5 trials on day 1 , and 3 trials on day 2 . The starting locations did not change during all training sessions and intertrial time intervals represented approximately $10 \mathrm{~min}$. Sixty min after the last trial on each day, a probe test was performed: the platform was removed, and the time spent in the quadrant of the original platform location was measured with a cut-off time of $30 \mathrm{~s}$. On day $4,48 \mathrm{~h}$ after the last training session, the animals were tested for retention. The platform was removed, each rat was again placed at the starting point, observed for $60 \mathrm{~s}$ and the time spent in the quadrant of the original platform location was recorded.

\subsection{Tissue collection and preparation}

After the end of behavioral experiments, the rats were decapitated. Brains were removed out of sculls and thoroughly washed in ice-cold isotonic saline solution. Cortical tissue (samples containing frontal, parietal, temporal and occipital cortex) were dissected on ice and immediately frozen in liquid nitrogen. All samples were stored at $-86^{\circ} \mathrm{C}$ until use. 


\subsection{Assessment of nerve growth factor (NGF) and brain-derived neurotrophic factor $(B D N F)$ in brain samples}

\subsubsection{Enzyme-linked immunosorbent assay (ELISA)}

ELISA was used to determine the amount of NGF and BDNF protein in brain extracts. We used commercially available kits: ChemiKine Nerve Growth Factor Sandwich ELISA kit and ChemiKine BrainDerived Neurotrophic Factor Sandwich ELISA kit (both Millipore, USA). All sample treatments and measurements were performed according to the manufacturers' protocol.

\subsubsection{Western-blot analysis}

Western-blot was also used for assessment of protein levels of NGF and BDNF and their receptors in the brain extracts. The supernatants were mixed with Laemmli buffer consisting of $62.5 \mathrm{mM}$ Tris- $\mathrm{HCl}$ (pH 6.8), $2 \%$ SDS, $10 \%$ glycerol, $0.001 \%$ bromophenol blue, and 5\% 2-mercaptoethanol and incubated at $95^{\circ} \mathrm{C}$ for $5 \mathrm{~min}$. The samples containing $50 \mathrm{pg}$ of protein were applied to $10 \%$ SDS-polyacrylamide gel. Electrophoresis was performed using Protean II xi Cell Bio-Rad device (Bio-Rad, USA). After the separation, proteins were transmitted onto the polyvinylidene difluoride (PVDF) membrane (BioRad, USA) using Fast Semi-Dry Blotter chamber (Thermo Scientific, USA). Non-specific binding was blocked using 5\% skimmed milk in a buffer consisting of $25 \mathrm{mM}$ Tris- $\mathrm{HCl}$ (pH 7.5), $150 \mathrm{mM} \mathrm{NaCl}, 0.1 \%$ Tween-20. Then, the membranes were incubated overnight at $4{ }^{\circ} \mathrm{C}$ in the blocking buffer containing one of the following antibodies: anti-Trk A (R\&D Systems, AF1056, 1:2000); anti- Trk B (R\&D Systems, MAB397, 1:2000); anti-p75 NGF (Abcam, ab38335, 1:5000); anti-NGF (H-20) (sc-548, Santa Cruz Biotechnology, 1:2000); anti-BDNF(ab72439, Abcam, 1:1000); or anti- $\alpha$-Tubulin (T9026, Sigma, $1: 10000)$. After washing, the membranes were incubated with the horseradish peroxidase-conjugated secondary antibodies (Bio-Rad, 1:1000). Binding of the secondary antibodies was detected using the SuperSignal West Femto chemiluminescence system (Thermo Scientific, USA) and RetinA X-ray film (Retina, Germany). Densitometry of protein bands was performed using Image J software (NIH, USA). The intensity of the target proteins was divided by the intensity of the $\alpha$-Tubulin as a loading control.

\subsection{Statistical analysis}

Statistical analysis was performed using Statistica for Windows 8.0 software. Student's $t$-test was used for comparison of data between the groups. Analysis of variances (ANOVA) with repeated measures and $\chi^{2}$ test were used to analyze the data of place learning in the water-maze. ANOVA was used for analysis of most data sets, while Mann-Whitney U-test was used to compare groups of data not fitting into normal distribution. Data are presented as $M \pm$ S.E.M.

\section{Results}

\subsection{Effects of CBL treatment on body weight in young and old rats}

At the start of the experiment ages of each mixed population of rats were 2-3, and 23-24 months. Each experimental series continued for about 1 month, however, we will use the above "initial" ages for indicating respective groups.

Prior to experimental treatment, the animals were tested in the open field (OF) test in order to estimate the initial levels of locomotor and exploratory activity and to randomize them into groups with similar activities. Averaged data from the OF testing in the young rat of Control (vehicle) and CBL treated groups ( $n=20$ in both groups) were 83.25 \pm 4.6 and $83.65 \pm 6.0$ squares crossed and $21.05 \pm 1.5$ and $18.65 \pm 1.5$ rearing, respectively. In the aged rats numbers of squares crossed were $70.7 \pm 6.7$ and $63.1 \pm 4.3$ and numbers of rearing were $19.8 \pm 2.0$ and $17.5 \pm 1.6$ in the Control $(n=17)$ and CBL $(n=18)$ treated groups, respectively. The average locomotor and exploratory activity as well as body weight were not different in Control vs CBL groups.

At the beginning of the experiment, in the Control group of young rats the average body weight was $260.0 \pm 16.2 \mathrm{~g}$ and in the CBL-treated young group the average body weight was $271.5 \pm 20.2 \mathrm{~g}$. After the last saline or CBL injections, the body weights were $354.7 \pm 30.8$ and $364.4 \pm 26.2 \mathrm{~g}$ in the Control and CBL groups, respectively. We observed a gradual increase in the body weight $(\mathrm{F}(19,703)=405.28$, $p<0.0001$; ANOVA with repeated measures) during the experiment. Treatment of rats with either vehicle (Control) or CBL did not significantly influence the body weight gain $(\mathrm{F}(1,37)=0.12, p=0.73)$, indicating that it did not depend on the type of treatment. 
Initially, in the Control group of old rats the average body weight was $684.1 \pm 20.4 \mathrm{~g}$ and in the CBL-treated group the average body weight was $653.2 \pm 26.2 \mathrm{~g}$. After the last injections, the respective body weights were $645.7 \pm 20.9$ and $614.3 \pm 28.1 \mathrm{~g}$ in the Control and CBL groups, respectively. Treatment of rats with either vehicle or CBL resulted in a gradual decrease in the body weight $(F(19,665)=16.12, p<0.0001$; ANOVA with repeated measures) and this effect did not depend on the type of treatment.

Thus, treatment with CBL at the dose of $2.5 \mathrm{ml} / \mathrm{kg}$ did not significantly influence general appearance of rats in any age population, estimated by body weight change, drinking and feeding behavior, color and state of the coat. During the course of the experiment we observed a gradual body weight gain in young rats and a minimal gradual body weight loss in old rats. We did not observe any effect of CBL treatment on this index in any age population of the animals.

\subsection{Effects of CBL treatment on place learning in a water maze and long-term memory in young and old rats}

Place learning in a water maze was used for assessment of learning and memory in young and old rats. The training was performed according to a four-day protocol described in Methods section and started one day after the OF test. During Day 1, the animals of vehicle-treated (Control) $(n=15)$ and CBL-treated $(n=15)$ groups of 2-3-month-old rats as well as vehicle-treated (Control) $(n=14)$ and CBL-treated $(n=14)$ groups of 23-24-monthold rats were trained to find a hidden platform in a rectangular pool. The data presented in Fig. 1A demonstrate a gradual decrease in the duration of escape latency in all four experimental groups $(\mathrm{F}(4$, $216)=45.5, p<0.001$; ANOVA with repeated measures). ANOVA revealed a significant effect of age $(\mathrm{F}(1,54)=24.2, p<0.001)$ and age $\times$ trial interaction $(\mathrm{F}(4,216)=2.71, p<0.05)$. However, no significant effect of treatment $(\mathrm{F}(1,54)=0.97, p=0.32)$, treatment $\times$ trial and age $\times$ treatment $\times$ trial interactions $(\mathrm{F}(4,216)=1.02, p=0.42$ and $\mathrm{F}(4,216)=0.96$, $p=0.43$, respectively) were found. These data may indicate a difference in the rate of training between the young and aged animals. Furthermore, we did not find any effect of age or treatment in the Probe trial 1 performed 60 min after the end of the training session on Day 1 in order to test the retention of platform location (Figs. 1B, C). Analysis of learning curves on Day 2
(Fig. 1A) revealed an effect of training on the escape latency $(\mathrm{F}(2,108)=5.33, p<0.01)$ again, though no effects of other factors or interactions between them were observed. We did not reveal any effect of age or treatment in the Probe trial 2, performed 60 min after the end of training session on Day 2 (Figs. 1B, C). Forty-eight hours after the last training session, the additional probe trial did not reveal difference in the time spent in the target quadrant between the two groups of animals studied (Figs. 1B, C).

However, a detailed analysis of training revealed that most obvious differences between the vehicleand CBL-treated groups of aged animals were observed during the first trial of Day 1 training session. Specifically, 50\% of control animals (7 of 14 rats) could not locate the hidden platform in this trial whereas in the CBL-treated group only $14 \%$ of animals (2 of 14 rats) exhibited inability to find the platform (Fig. 1A insert). This difference was significant according to the $\chi^{2}$ test $\left(\chi^{2}=4.09, \mathrm{df}=1\right.$, $p<0.05)$. In the fifth trial of Day 1 , all animals of the CBL-treated group successfully located the platform while 1 of 14 rats of the control age-matched group could not perform the task. In the first trial of Day 2, all CBL-treated rats successfully performed the task while 2 control age-matched animals could not find the hidden platform. A different pattern of task performance was observed in 2-3-month-old rats. During the first trial of Day 1 training, $26 \%$ of control animals (4 of 15 rats) and $40 \%$ of CBL-treated animals (6 of 15 rats) could not locate the hidden platform, this difference being insignificant $\left(\chi^{2}=0.60, \mathrm{df}=1, p=0.44\right)$. At the end of training on Day 1, all animals exhibited short latencies to find the platform. Three-month-old animals of both groups exhibited similar capabilities to perform the task during the first trial of Day 2.

Thus, animals of the two age groups exhibited different efficacy of training in the simplified version of the water maze task. Only minor effect of CBL was observed in old rats at the start of training and no effect on memory retention was found.

\subsection{Effects of CBL treatment on the contents of $N G F$ and BDNF in the neocortex of young and old rats}

We have used two different approaches to assess the expression of NGF and BDNF in the neocortex. The expression of neurotrophins was evaluated by the sandwich ELISA method, using commercially available reagent kits, and the Western blot (WB) method, using available antibodies. 

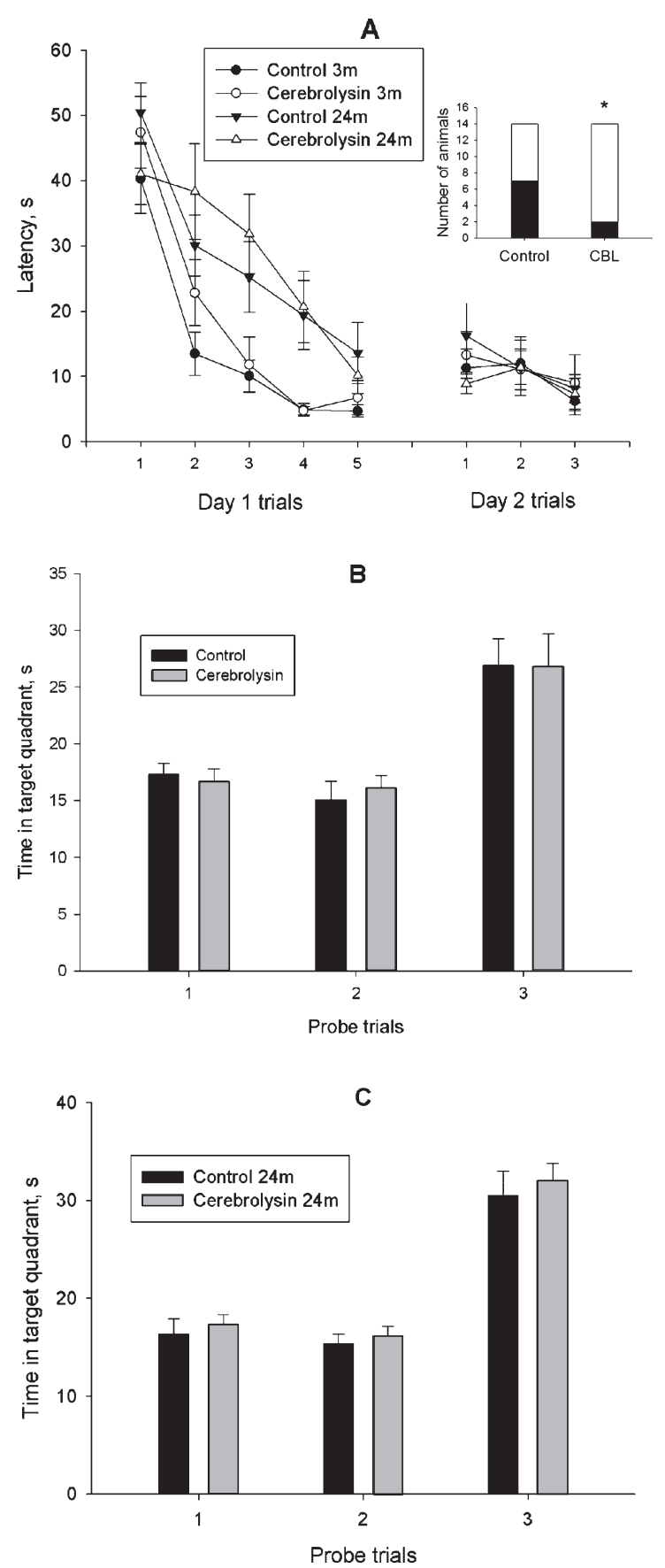

Fig. 1. Effect of CBL on the place learning in the water-maze test in 2-3- and 23-24-month-old rats. A, mean latency to reach a hidden platform; A insert, number of 23-24-month-old animals, which reached a hidden platform in the trial 1 of training Day 1 . White part of the bar represents the number of animals, which reached the platform; $\mathrm{B}$ and $\mathrm{C}$, data on the probe trials 1 and 2 performed on training Days 1 and 2, respectively and probe trial 3, performed $48 \mathrm{~h}$ after training. $N=14-15$ per group. Data in A-C are presented as $M \pm$ S.E.M. * - $p<0.05$ difference between Control and CBL-treated groups according to $\chi^{2}$ test.

\subsubsection{Total expression of $N G F$ and BDNF (ELISA)}

Data in Fig. 2A demonstrate the expression of NGF in the neocortex of rats of different age groups and the effects of course CBL treatment on the expression of this neurotrophin. Total NGF expression was higher in the neocortex of old rats as compared to young ones; however, CBL treatment did not affect it at either age. In contrast to NGF, BDNF expression was not significantly different between young and old rats. Though the data presented in Fig. 2B might suggest that the BDNF levels were lower in the aged rats, the difference between young and old animals was not statistically significant, and did not even show a statistical trend $(P>0.1)$. Similarly to NGF, CBL course did not affect the levels of BDNF (Fig. 2B).
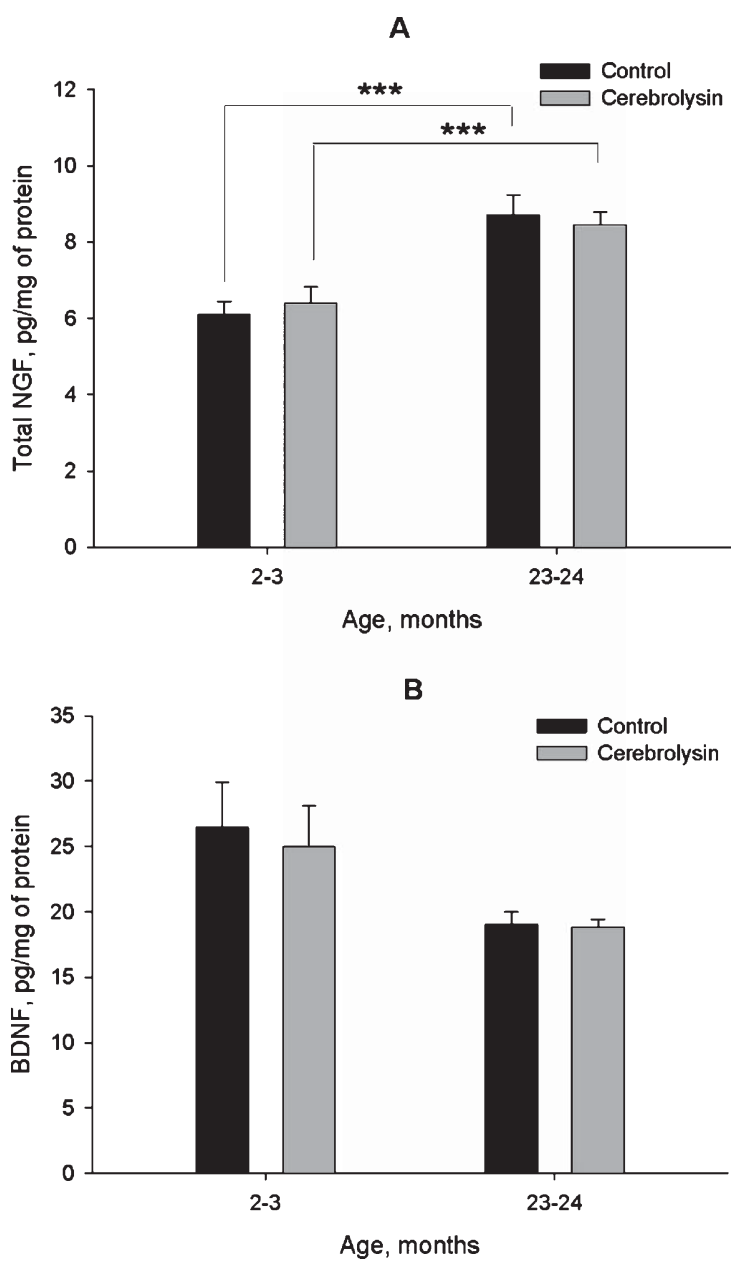

Fig. 2. Effect of CBL on NGF (A) and BDNF (B) levels in the neocortex of young and old rats. NGF and BDNF levels were measured using ELISA. Vehicle $(n=10-12$ per group), CBL $(n=7-12)$. Data are presented as $M \pm$ S.E.M. *** - $p<0.001$ Newman-Keuls test. 


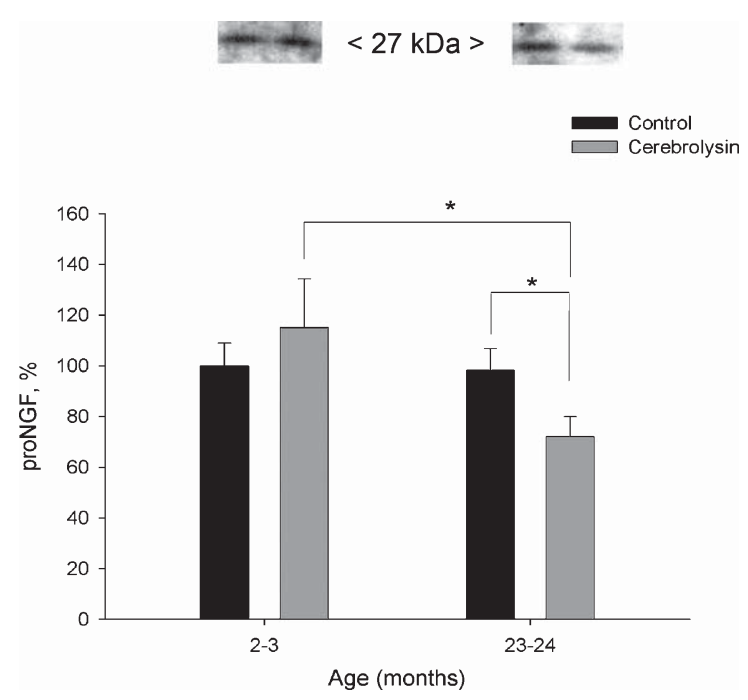

Fig. 3. Effect of CBL on pro-NGF expression in the neocortex of young and old rats. pro-NGF content was measured using WB. Vehicle ( $n=7-8$ per group), CBL $(n=7-8)$. Data are presented as $M \pm$ S.E.M. * $-p<0.05$ versus old control and respective young group, Mann-Whitney U-test.

\subsubsection{Expression of NGF and BDNF proforms (WB)}

Expression of pro-NGF in the neocortex was not significantly affected by aging (Fig. 3). CBL course effectively decreased proNGF in the neocortex of old animals almost 1.4 fold as compared with the respective age control. The level of pro-NGF in this group was also significantly lower as compared to 2-3-month-old CBL-treated rats. In the neocortex, proBDNF expression did not show statistically significant changes with age; neither did it demonstrate significant influence of CBL course (data not shown).

\subsection{Effects of CBL treatment on expression of neurotrophin receptors in the neocortex of young and old rats}

\subsubsection{TrkA receptors}

Using anti-TrkA antibodies in Western blots, two forms of TrkA could be detected, a partially glycosylated TrkA precursor and completely glycosylated mature TrkA. These two proteins could be detected as bands corresponding to molecular weights of 110 and $140 \mathrm{kDa}$, respectively. In the neocortex, the glycolsylated TrkA gp140 expression decreased with age (2.3 times from 2-3 to 23-24 months; Fig. 4). CBL significantly improved the expression of TrkA gp140 in old animals (1.5 times; Fig. 4), though, the level of TrkA gp140 still remained lower as compared to the respective group of young animals. The expression

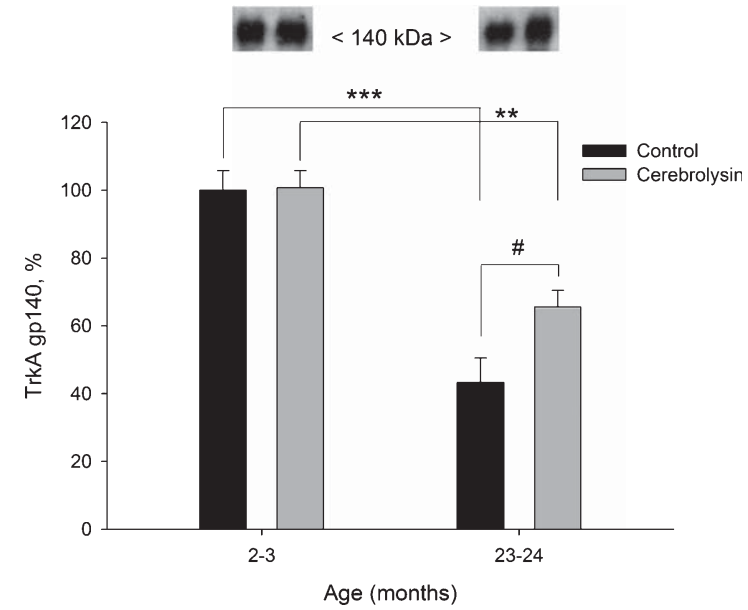

Fig. 4. Effect of CBL on TrkA gp140 expression in the neocortex of young and old rats. TrkA gp140 content was measured using WB. Vehicle ( $n=7-8$ per group), CBL $(n=7-8)$. Data are presented as $M \pm$ S.E.M. $* *, * * *-p<0.01, p<0.001$ versus respective young groups; \# - $p<0.05$ versus respective age control, MannWhitney U-test.

of the partially glycosylated precursor TrkA gp1 10 in the neocortex was not significantly affected by either age or CBL treatment (data not shown).

\subsubsection{Trk B receptors}

Using anti-TrkB antibodies in Western blots, two protein bands of TrkB could be detected: a complete and active form and truncated and inactive form. These two proteins could be distinguished as bands corresponding to molecular weights of 145 and 95 $\mathrm{kDa}$, respectively. We observed an age-dependent decrease in the expression of the active form of the receptor, TrkB gp145 in the neocortex (Fig. 5). The expression of TrkB gp145 was 1.6 times lower in old rats as compared to young animals. CBL did not influence neocortical TrkB gp145 significantly.

The expression of the truncated and presumably inactive TrkB gp95 in the neocortex was not significantly affected by either age or CBL treatment (data not shown).

\subsection{3. $p 75 N T R$}

Expression of the p75 neurotrophin receptor protein was measured using anti-p75NTR antibodies. We observed a strong age-related decrease in the expression of p75NGF in the neocortex; a two-fold decline was found in old rats as compared to young animals (Fig. 6). CBL treatment significantly decreased the p75NGF expression (1.5 times) in 2-3-monthold animals and significantly increased the p75NGF expression (1.5 times) in 23-24-month-old rats. 


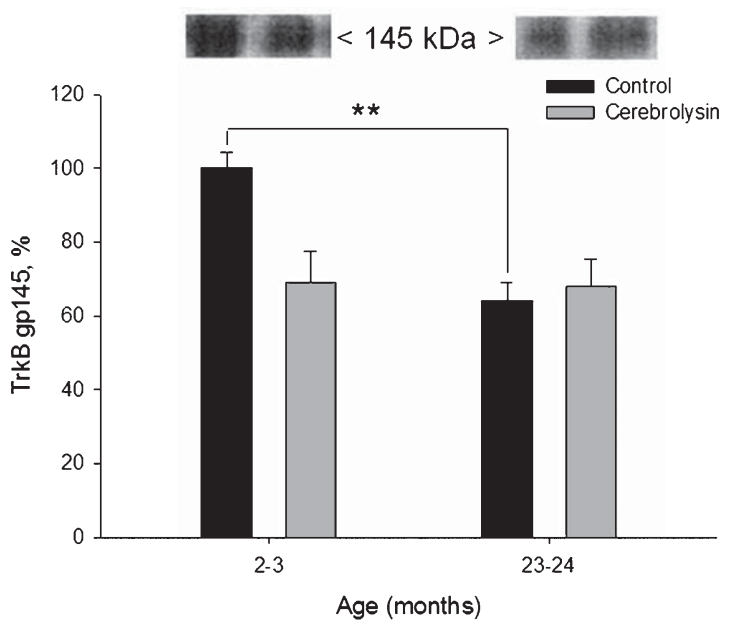

Fig. 5. Effect of CBL on TrkB gp145 expression in the neocortex of young and old rats. TrkB gp145 content was measured using WB. Vehicle ( $n=7-8$ per group), CBL $(n=7-8)$. Data are presented as $M \pm$ S.E.M. ${ }^{* *}-p<0.01$ versus respective young group, MannWhitney U-test.

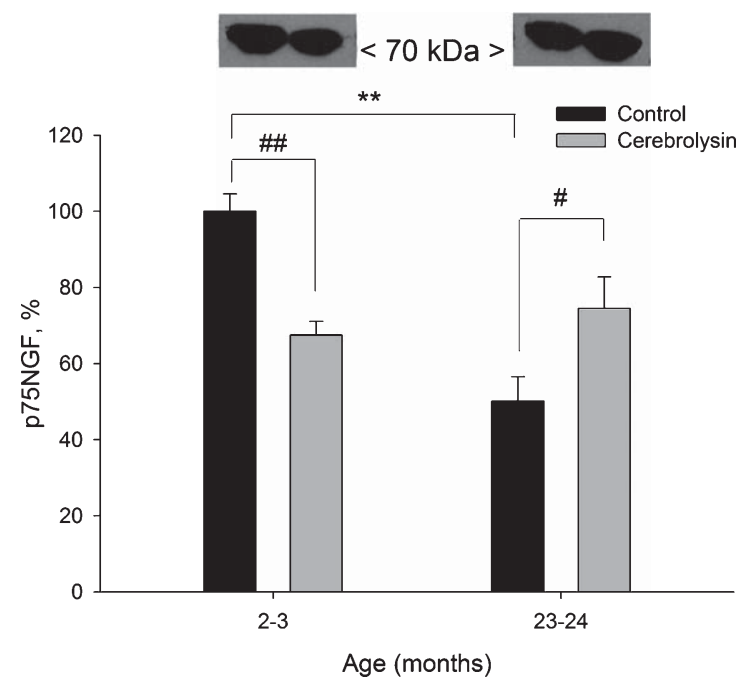

Fig. 6. Effect of CBL on p75NGF expression in the neocortex of young and old rats. Vehicle ( $n=7-8$ per group), CBL $(n=7-8)$. Data are presented as $M \pm$ S.E.M. $* *$ - $p<0.01$ versus respective young group; \#, \#\# - $p<0.05$ and $p<0.01$, respectively, versus respective age control, Mann-Whitney U-test.

\section{Discussion}

In our study, CBL treatment was well tolerated by both young and old Wistar rats. It did not affect general status, age-related weight gain/loss, and general locomotor activity. As expected, in the present study young rats learned more rapidly place of locating a hidden platform in the simplified water maze procedure (taking into account learning curves on Day 1) as compared to old rats. The animals of both young groups successfully located a hidden platform in all probes and retention trials. However, a minor though statistically significant effect of CBL treatment course was evident in old rats: the experimental treatment improved learning in old rats as assessed by a higher portion of animals capable to locate a platform in a water maze in the first trial of Day 1 training. Remarkably, this difference was transient since animals of both CBL- and vehicle-treated groups successfully located the platform in the retention test. Thus, we did not find any substantial effect of CBL on learning and memory retention in healthy young and old animals. Gschanes and Windisch (1998) studied the effects of CBL course $(2.5 \mathrm{ml} / \mathrm{kg}$, i.p., for 19 days) in 24-month-old rats tested in a standard Morris water maze. The authors demonstrated that CBL treatment improved the spatial learning and memory of old male rats. Interestingly, female rats displayed an even greater response. Hutter-Paier et al. (1996) demonstrated that after a 7-day pretreatment of 24- month-old rats with CBL the animals displayed better performance in a step-through avoidance task. CBL-treated 24-month-old rats displayed increased GluR1 density in the hippocampal formation (Eder et al., 2001), increase in GLUT1 (Gschanes et al., 2000) as well as an enhancement in the number of synaptophysin-immunostained presynaptic terminals in the entorhinal cortex, and hippocampus (Reinprecht et al., 1999); these modifications correlated with beneficial cognitive effects following CBL therapy. Although our data confirm earlier results related to effects of CBL on learning and memory of normal old rodents, this effect was less expressed in this study probably because of relatively higher simplicity of the task paradigm used.

It is well-known that the predominant proportion of the neurotrophin pool in the brain is represented by their pro-form. Using respective antibodies for Western blotting we did not observe any age-related dynamics in proNGF or proBDNF in the neocortex (Fig. 3). However, an ELISA- based readout, reported to detect both pro- and mature forms of neurotrophins, showed significant age dynamics for NGF in the neocortex (Fig. 2). Interestingly, NGF expression in the neocortex increased in old rats, which is in line with data reported earlier by other groups (Bimonte-Nelson et al., 2008; Perovic et al., 2013; Terry Jr. et al., 2011).

To complement the analysis of age-dependent dynamics in neurotrophin levels, we investigated 
their respective receptors, aiming to further understand the age-related changes in neurotrophin system and potential effects of CBL. The interpretation of study results are additionally complicated by various Trks species on Western blots, differing in their glycosylation levels, and their resulting molecular weight. In addition, most of the available data on direct Trk evaluation were derived from cell cultures studies, and not from nervous tissue, as it was analyzed in our study. An $80 \mathrm{kDa}$ polypeptide is regarded as the non-glycosylated backbone of TrkA. Glycosylation of this backbone results in a partially glycosylated gp110, a glycoprotein which is further glycosylated to yield gp140. Both, gp110 and gp140, have similar Trk activities in vitro, but this may not reflect their in vivo activities (Martin-Zanca et al., 1989; 1990). Indeed, TrkA glycosylation was shown to prevent ligand independent activation and it is fundamental for plasma membrane localization of TrkA, for activation of the Ras/MAPK pathway and subsequent induction of neuronal differentiation in PC12 cells (Watson et al., 1999; Bucci et al., 2014). In our experiments, a significant age-dependent decrease in active, maximally glycosylated forms of TrkA (140 kDa) and TrkB (145 kDa) was revealed in the neocortex (Figs. 4 and 5), while no major changes of partially glycosylated forms (110kDa and $95 \mathrm{kDa}$, respectively) could be detected. Age-related changes in neocortical p75NTR receptor were similar to those of Trk receptors. Taken together, aging induced a concerted decrease in neurotrophin receptors in the neocortex.

Neurotrophic factors are considered to be part of the therapeutic strategy for neurological disorders, in particular dementia, stroke and traumatic brain injury. CBL is a neuropeptide preparation which shows proneuroplastic pharmacological effects reminiscent of the action of endogenous neurotrophic factors with respect to neuroprotection and repair (Bornstein \& Poon, 2012; Chen et al., 2013a; 2013b; Muresanu et al., 2015; 2016). In the presented study, changes in neurotrophin expression induced by CBL were not dramatic, which could be explained by the use of a non-pathological animal model. However, CBL treatment seemed to influence the neocortical neurotrophin system in the context of aging. In general the observed CBL effects appeared to counteract the changes induced by the aging process. Three significant effects deserve attention and appear to be of functional importance.

First, we demonstrated a significant CBL-induced decrease in pro-NGF expression in the neocortex of old animals. Immunoblot analysis performed by
Ubhi et al. (2013) demonstrated that CBL treatment of transgenic mice, expressing the human amyloid precursor protein (hAPP), resulted in lowered levels of pro-NGF when compared to saline-treated control animals. Our result of CBL- mediated decrease of pro-NGF is consistent with the data of Ubhi et al. (2013), however, for technical reasons, we could not show increased mature NGF or total NGF immunoreactivity in our experimental set up, as it was shown in hAPP tg mice. Yet, the levels of total NGF remained unaffected by the treatment (Fig. 2) suggesting that the changes observed for pro-NGF levels (Fig. 3) may reflect a shift in the ratio of pro- and mature-NGF as observed by Uhbi et al. (2013).

Second, we have shown an increase in the fully glycosylated active $140 \mathrm{kDa}$ TrkA receptor in aged animals upon treatment with CBL and, importantly, CBL treatment mediated a reversal of the age-related drop in $140 \mathrm{kDa}$ TrkA expression in the neocortex of old animals.

Third, we revealed a similar effect of CBL on p75NTR receptor, namely the reversal of its agerelated decrease in the neocortex of old animals. Many studies have been published in support of either death or survival functions of the p75 neurotrophin receptor. p75NTR has been generally regarded as a receptor mediating cell death, in contrast to Trks which are thought to promote cell survival. However, recent data demonstrated that the p75NTR receptor is also involved in regulation of neural plasticity in the mature nervous system, in promotion of adult neurogenesis and that it is increasingly expressed in neurons and glial cells in response to injury increasing neurotrophin affinity of Trks (see Meeker and Williams, 2015 for review). Having no intrinsic catalytic activity, p75NTR interacts with and modulates the function of TrkA, TrkB, and TrkC, as well as sortilin and the Nogo receptor, thereby providing substantial cellular and molecular diversity for regulation of neuron survival, neurogenesis, immune responses and processes that support neural function. It is suggested that upregulation of p75NTR under pathological conditions reflects its key position to control numerous processes underlying nervous system recovery. Thus, the observed decrease in p75NTR expression in the neocortex of aged rats in our study could be interpreted as impairment in the compensatory potential of the old brain. Consequently, CBL's effect on the increase of p75NTR expression in the old brain to a level that is more comparable with the young animals is indicative of the anti-aging effect of the peptidergic drug. 
Taken together our data suggest that CBL may have specific age-dependent features. In the old brain with a relative neurotrophic factor deficit, CBL's action appears to counteract specific processes associated with aging, i.e. by restoring molecular determinants to levels seen in younger animals, as demonstrated for TrkA and p75NTR.

Surprisingly, in young animals CBL treatment led to the opposite outcome as compared to old animals, namely the decrease of p75NTR. Importantly the overall status on the animals as monitored by animal behavior and body weight was not affected by CBL treatment. In the young brain, neurotrophic capacity is relatively high and introduction of an additional potent external neurotrophic signal, such as CBL, might "silence" some parallel neurotrophic pathways. p75NTR interacts with all known neurotrophin receptors, TrkA, TrkB, TrkC, and each of these p75NTR -mediated processes is thought to contribute in different ways to fine tuning of a wide range of cellular functions supporting the development, maturation and maintenance of the nervous system (Kraemer et al., 2014). Thus, modulation of p75 NTR may prevent excessive neurotrophic signaling unnecessary in the young brain.

Several studies on CBL showed that this peptide preparation is mimicking the action of neurotrophic factors; however, the exact mode of action of CBL in the pathological context of aging remains elusive. Evidence points to the fact that CBL is able to affect signaling pathways which can modify neurotrophic factors systems. Here we present the first direct evidence for a beneficial influence of CBL course on neurotrophin system of the aged brain of rodents. CBL specifically affects the neocortical NGF system.

\section{Conclusion}

In this study we show that aging is accompanied by changes in neurotrophin system in the neocortex: a decrease in maximally glycosylated forms of TrkA (140 kDa) and TrkB (145 kDa), as well as p75NTR expression. In old animals, CBL course $(2.5 \mathrm{ml} / \mathrm{kg}, 20$ injections) slightly but significantly improved long-term memory, decreased the expression of proNGF and counteracted effects of aging on TrkA, and p75NTR expression in the neocortex. These data substantiate the concept that the anti-aging effects of CBL are associated with the pro-neuroplastic modulation of the neocortical NGF system. However, we observed only minor effects of
CBL treatment on behavior of young and aged animals. Furthermore, the "rejuvenating" effect of CBL on the NGF system in the brain of aged rats did not correlate with improvement of their learning and memory capacities. It is possible that the age-related alterations in learning capacities of older animals are within the normal range of changes in adaptive behavior. It would be too simple to suggest that age-related changes in neurotrophin systems correspond one-toone to alterations in definite behavioral phenomena; even a clear shift in the indices of these systems to the levels observed in younger animals could hardly unequivocally be reflected in all behavioral features. It is possible that more significant behavioral effects of CBL treatment would be observed in the animals with cerebral pathologies, as it has been observed in a mouse model of Alzheimer's disease (Ubhi et al., 2013). No doubt that further experimental investigation is needed, but the data of the present study provide evidence that CBL holds the potential as an anti-aging agent.

\section{Acknowledgments}

This work was partly funded by EVER NeuroPharma $\mathrm{GmbH}$ and Russian Science Foundation (grant \# 14-25-00136; studies on receptors of neurotrophins).

\section{References}

Bimonte-Nelson, H.A., Granholm, A.C., Nelson, M.E., \& Moore, A.B. (2008). Patterns of neurotrophin protein levels in male and female Fischer 344 rats from adulthood to senescence: How young is "young" and how old is "old"? Experimental Aging Research, 34(1), 13-26.

Bornstein, N. \& Poon, W.S. (2012). Accelerated recovery from acute brain injuries: Clinical efficacy of neurotrophic treatment in stroke and traumatic brain injuries. Drugs Today (Barcelona), 48(suppl. A), 43-61.

Bothwell, M. (2014). NGF, BDNF, NT3, and NT4. Handbook of Experimental Pharmacology, 220, 3-15.

Bucci, C., Alifano, P. \& Cogli, L. (2014). The role of rab proteins in neuronal cells and in the trafficking of neurotrophin receptors. Membranes (Basel), 4(4), 642-677.

Budni, J., Bellettini-Santos, T., Mina, F., Garcez, M.L. \& Zugno, A.I. (2015). The involvement of BDNF, NGF and GDNF in aging and Alzheimer's disease. Aging and Diseases, 6(5), 331-341.

Chao, M.V., Rajagopal, R. \& Lee, F.S. (2006). Neurotrophin signaling in health and disease. Clinical Science (London), $110(2), 167-173$. 
Chen, C.C., Wei, S.T., Tsaia, S.C., Chen, X.X. \& Cho, D.Y. (2013a). Cerebrolysin enhances cognitive recovery of mild traumatic brain injury patients: Double-blind, placebo-controlled, randomized study. British Journal of Neurosurgery, 27(6), 803-807.

Chen, N., Yang, M., Guo, J., Zhou, M., Zhu, C. \& He, L. (2013b). Cerebrolysin for vascular dementia. Cochrane Database Systematic Reviews, 1, CD008900.

Crutcher, K.A. \& Weingartner, J. (1991). Hippocampal NGF levels are not reduced in the aged Fischer 344 rat. Neurobiology of Aging, 12(5), 449-454.

Cuello, A.C., Bruno, M.A. \& Bell, K.F. (2007). NGF-cholinergic dependency in brain aging, MCI and Alzheimer's disease. Current Alzheimer Research, 4(4), 351-358.

Eder, P., Reinprecht, I., Schreiner, E., Skofitsch, G. \& Windisch, M. (2001). Increased density of glutamate receptor subunit 1 due to Cerebrolysin treatment: An immunohistochemical study on aged rats. Histochemical Journal, 33(11-12), 605-612.

Gschanes, A. \& Windisch, M. (1998). The influence of Cerebrolysin and E021 on spatial navigation of 24-month-old rats. Journal of Neural Transmission Suppl, 53, 313-321.

Gschanes, A., Boado, R., Sametz, W. \& Windisch, M. (2000). The drug cerebrolysin and its peptide fraction E021 increase the abundance of the blood-brain barrier GLUT1 glucose transporter in brains of young and old rats. Histochemical Journal, 32(2), 71-77.

Hutter-Paier, B., Eggenreich, U. \& Windisch, M. (1996). Effects of two protein-free peptide derivatives on passive avoidance behaviour of 24-month-old rats. Arzneimittelforschung, 46(3), 237-241.

Kelley, A.E. (1993). Locomotor activity and exploration. Behavioural Neuroscience A Practical Approach, vol. II, New York: IRL Press, pp. 1-21.

Kraemer, B.R., Yoon, S.O. \& Carter, B.D. (2014). The biological functions and signaling mechanisms of the $\mathrm{p} 75$ neurotrophin receptor. Handbook of Experimental Pharmacology, 220, 121-164.

Lapchak, P.A., Araujo, D.M., Beck, K.D., Finch, C.E., Johnson, S.A. \& Hefti, F. (1993). BDNF and trkB mRNA expression in the hippocampal formation of aging rats. Neurobiology of Aging, 14(2), 121-126.

Martin-Zanca, D., Barbacid, M. \& Parada, L.F. (1990). Expression of the trk proto-oncogene is restricted to the sensory cranial and spinal ganglia of neural crest origin in mouse development. Genes and Development, 4(5), 683-694.

Martin-Zanca, D., Oskam, R., Mitra, G., Copeland, T. \& Barbacid, M. (1989). Molecular and biochemical characterization of the human trk proto-oncogene. Molecular and Cellular Biology, 9(1), 24-33.

Masliah, E. \& DIez-Tejedor, E. (2012). The pharmacology of neurotrophic treatment with Cerebrolysin: Brain protectionand repair to counteract pathologies of acute and chronic neurological disorders. Drugs Today (Barcelona), 48(suppl. A), 3-24.
Maurice, T., Lockhart, B.P. \& Privat, A. (1996). Amnesia induced in mice by centrally administered beta-amyloid peptides involves cholinergic dysfunction. Brain Research, 706(2), 181-193.

Meeker, R.B. \& Williams, K.S. (2015). The p75 neurotrophin receptor: At the crossroad of neural repair and death. Neural Regeneration Research, 10(5), 721-725.

Muresanu, D.F., Ciurea, A.V., Gorgan, R.M., Gheorghita, E., Florian, S.I., H. Stan,... \& Alvarez, A. (2015). A retrospective, multi-center cohort study evaluating the severity- related effects of cerebrolysin treatment on clinical outcomes in traumatic brain injury. CNS and Neurological Disorders - Drug Targets, 14(5), 587-599.

Muresanu, D.F., Heiss, W.D., Hoemberg, V., Bajenaru, O., Popescu, C.D., Vester, J.C. ...\& Guekht, A. (2016). Cerebrolysin and recovery after stroke (CARS): A randomized, placebo-controlled, double-blind, multicenter trial. Stroke, 47(1), 151-159.

Niewiadomska, G., Mietelska-Porowska, A. \& Mazurkiewicz, M. (2011). The cholinergic system, nerve growth factor and the cytoskeleton. Behavioural Brain Research, 221(2), 515-526.

Perovic, M., Tesic, V., Mladenovic Djordjevic, A., Smiljanic, K., Loncarevic-Vasiljkovic, N., Ruzdijic, S. \& Kanazir, S. (2013). BDNF transcripts, proBDNF and proNGF, in the cortex and hippocampus throughout the life span of the rat. Age (Dordrecht), 35(6), 2057-2070.

Reinprecht, I., Gschanes, A., Windisch, M. \& Fachbach, G. (1999). Two peptidergic drugs increase the synaptophysin immunoreactivity in brains of 24-month-old rats. Histochemical Journal, 31(6), 395-401.

Shi, S.S., Shao, S.H., Yuan, B.P., Pan, F. \& Li, Z.L. (2010). Acute stress and chronic stress change brain-derived neurotrophic factor (BDNF) and tyrosine kinase-coupled receptor (TrkB) expression in both young and aged rat hippocampus. Yonsei Medical Journal, 51(5), 661-671.

Terry Jr., A.V., Kutiyanawalla, A. \& Pillai, A. (2011). Agedependent alterations in nerve growth factor (NGF)-related proteins, sortilin, and learning and memory in rats. Physiology and Behavior, 102(2), 149-157.

Ubhi, K., Rockenstein, E., Vazquez-Roque, R., Mante, M., Inglis, C., Patrick, C. .. \& \& Masliah, E. (2013). Cerebrolysin modulates pronerve growth factor/nerve growth factor ratio and ameliorates the cholinergic deficit in a transgenic model of Alzheimer's disease. Journal of Neuroscience Research, 91(2), 167-177.

Ukraintseva, S.V., Arbeev, K.G., Michalsky, A.I. \& Yashin, A. I. (2004). Antiaging treatments have been legally prescribed for approximately thirty years. Annals of the New-York Academy of Sciences, 1019, 64-69.

Watson, F.L., Porcionatto, M.A., Bhattacharyya, A., Stiles, C.D. \& Segal, R.A. (1999). TrkA glycosylation regulates receptor localization and activity. Journal of Neurobiology, 39(2), 323-336. 